Revue de droit comparé du travail et de la sécurité sociale

3 | 2019

Les migrations internationales de travail

\title{
L'élargissement du cercle des allocataires des prestations familiales
}

Anna Aleksandrova

\section{(2) OpenEdition}

1 Journals

Édition électronique

URL : https://journals.openedition.org/rdctss/1520

DOI : $10.4000 /$ rdctss. 1520

ISSN : 2262-9815

Éditeur

Centre de droit comparé du travail et de la sécurité sociale

Édition imprimée

Date de publication : 1 novembre 2019

Pagination : 196-199

ISSN : 2117-4350

\section{Référence électronique}

Anna Aleksandrova, "L'élargissement du cercle des allocataires des prestations familiales », Revue de droit comparé du travail et de la sécurité sociale [En ligne], 3 | 2019, mis en ligne le 01 novembre 2021, consulté le 13 novembre 2021. URL : http://journals.openedition.org/rdctss/1520 ; DOI : https:// doi.org/10.4000/rdctss. 1520

\section{(c) (†) $९$}

Revue de droit comparé du travail et de la sécurité sociale est mise à disposition selon les termes de la Licence Creative Commons Attribution - Pas d'Utilisation Commerciale - Pas de Modification 4.0 International. 


\section{ANNA ALEKSANDROVA}

UNIVERSITÉ d'ÉTAT DE PENZA

\section{L'ÉLARGISSEMENT DU CERCLE DES ALLOCATAIRES DES PRESTATIONS FAMILIALES}

En juillet 2019, le Parlement russe a adopté la loi n³05 qui élargit le cercle des personnes ayant droit aux allocations familiales ${ }^{1}$. Promulguée par le Président de la Russie le 2 août 2019, cette loi entrera en vigueur le 1er janvier 2020. Elle prévoit la modification de la loi $n^{\circ} 418$ sur les allocations familiales mensuelles qui avait été adoptée le 28 décembre 2017 à l'initiative du Chef de l'Etat².

Afin de clarifier le contenu des modifications apportées, il convient d'examiner préalablement le contenu de la loi $n^{\circ} 418$, d'identifier sa place dans le système de réglementation législative des prestations familiales, ainsi que les conditions et la procédure de versement des allocations prévues.

\section{I - LE SYSTÈME DES PRESTATIONS FAMILIALES EN RUSSIE}

La Russie est un État fédéral composé de 85 Régions (sujets de la Fédération). Ainsi, la réglementation normative en matière de protection sociale est appliquée aux niveaux fédéral et régional. Selon la partie 1 de l'art. 72 de la Constitution de Russie de 1993, la protection sociale, y compris la sécurité sociale, est administrée conjointement par la Fédération et ses entités constitutives. À cet égard, les prestations familiales peuvent être fédérales ou régionales. La présente contribution est consacrée aux prestations familiales fédérales.

En Russie, le système de prestations offert aux personnes ayant des enfants (niveau fédéral) comprend:

- I'allocation aux femmes en couches, dénommée «allocation de grossesse et d'accouchement»;

- I'allocation forfaitaire aux femmes ayant déclaré leur grossesse avant la fin de la $12^{\text {ème }}$ semaine de grossesse ;

- l'allocation de maternité (prime de naissance);

- I'allocation forfaitaire pour l'adoption d'un enfant;

- l'indemnité mensuelle de congé parental (jusqu'aux 18 mois de l'enfant);

- les allocations aux familles de militaires (indemnité forfaitaire versée à la femme enceinte d'un militaire affecté au service militaire, allocation mensuelle pour un enfant de militaire affecté au service...);

1 Loi fédérale du 2 août 2019 n 305 portant modification de la Loi fédérale sur les allocations familiales mensuelles: http://www.garant.ru/hotlaw/federal/1286252/

2 Loi fédérale du 28 décembre $2017 n^{\circ} 418$ sur les allocations familiales mensuelles: http://www. consultant.ru/document/cons doc LAW 286470/ 
- les allocations compensatoires (compensation mensuelle de congé parental jusqu'aux 36 mois de l'enfant, compensation pour la pension alimentaire dans les établissements d'enseignement...);

- le « capital maternel»;

- I'allocation familiale mensuelle.

Il faut souligner que la législation russe en matière de sécurité sociale n'est pas codifiée, c'est-à-dire qu'il n'existe pas de document unique dénommé "Code », contrairement aux Code du travail, Code Civil, Code de la famille... existant en Russie. Auparavant, certains domaines de la sécurité sociale (allocations familiales, pensions) étaient entièrement réglementés par des actes législatifs majeurs dits «lois codifiées». Toutefois, au cours des vingt dernières années, de nombreuses modifications législatives et adoptions de nouvelles lois sont intervenues, entraînant de fait la « décodification » de la législation sociale ${ }^{3}$. Cette tendance est également observée pour la législation relative aux prestations familiales.

La plupart des prestations familiales susmentionnées - à l'exception des trois dernières ont été instaurées par la loi du 19 mai 1995 n 814; les allocations compensatoires étant pour leur part prévues par les actes réglementaires. En 2006, le législateur a introduit une nouvelle forme de prestation familiale, le « capital maternel », instaurée par l'adoption de la loi du 29 décembre $2006 n^{\circ} 256^{5}$ (et donc sans modifier la loi n 81 ).

Parmi les caractéristiques les plus importantes de l'allocation «capital maternel ${ }^{6}$, on relève que:

- le capital maternel est versé à la mère ou au père seul (ou dans des cas exceptionnels à l'enfant lui-même), en cas de naissance ou d'adoption d'un deuxième enfant ou d'un enfant suivant, si cette naissance (ou adoption) a eu lieu entre le $1^{\text {er }}$ janvier 2007 et jusqu'au 31 décembre 2021;

- il n'est versé qu'une seule fois;

- son montant est actuellement fixé à 453026 roubles (environ 6200 euros), mais le Gouvernement prévoit de l'augmenter de $4 \%$ en janvier $2020^{7}$ (soit environ 470000 roubles et 6440 euros);

- le capital maternel peut être obtenu sur la base d'un certificat délivré par le Fonds de pension de la Russie;

- en règle générale, il est attribué sous forme de virement et ne peut être versé en espèces (sauf exception examinée ci-après);

- I'utilisation du capital maternel est limitée à l'éducation des enfants, la constitution d'une épargne retraite (pour les femmes), l'amélioration des conditions de vie de la famille, l'achat de matériels et services pour la réinsertion sociale des enfants handicapés.

En 2017, à l'initiative du Président de la Russie, un nouveau type de prestations

3 E.G. Toutchkova, Le droit de la sécurité sociale de la Russie, Moscou, 2016, p. 83.

4 Loi fédérale du 19 mai $1995 n^{\circ} 81$ sur les prestations aux personnes ayant des enfants: http://www. consultant.ru/document/cons doc LAW 6659/

5 Loi fédérale du 29 décembre 2006 n ${ }^{\circ} 256$ sur les mesures supplémentaires de soutien aux familles avec enfants: http://www.consultant.ru/document/cons doc LAW 64872/

6 E. Serebryakova, Revue de droit comparé du travail et de la sécurité sociale, n²018/1, p. 157.

7 https://rg.ru/2019/06/06/reg-szfo/materinskij-kapital-budet-uvelichen-s-2020-goda.html 
familiales a été introduit: I'allocation familiale mensuelle. Dans ce cas, le législateur a pris la voie choisie précédemment (en 2006) en adoptant la nouvelle loi du 28 décembre 2017 $n^{\circ} 418$ sur les allocations familiales mensuelles.

Ainsi, aujourd'hui, au moins trois lois fédérales réglementent l'octroi des prestations familiales, ce qui semble constituer un défaut important de la réglementation juridique.

\section{II - CONDITIONS ET PROCÉDURE D'ATTRIBUTION DE L'ALLOCATION FAMILIALE MENSUELLE AVANT LA RÉFORME DE 2019}

L'allocation familiale mensuelle est versée aux allocataires russes résidant en Russie, sous réserve des conditions suivantes:

- naissance (ou adoption) du premier ou du deuxième enfant à partir du $1^{\text {er }}$ janvier 2018;

- l'enfant est citoyen de la Fédération de Russie;

- le quotient familial ne dépasse pas 1,5 fois le minimum vital de la population active (montant établi au niveau régional lors du $2^{\text {ème }}$ trimestre de l'année précédant l'année de la demande d'allocation).

L'allocation pour le premier enfant est versée à la mère, au père, au parent adoptif ou au tuteur de l'enfant. L'allocation pour le deuxième enfant est versée à la personne qui a obtenu le certificat au capital maternel (délivré par le Fonds de pension). En outre, la loi $n^{\circ} 418$ prévoit que l'allocation est versée jusqu'à ce que l'enfant atteigne 18 mois. Le montant de l'allocation est fixé au minimum vital de l'enfant établi au niveau régional pour le deuxième trimestre de l'année précédant l'année de la demande d'allocation.

Pour établir le minimum vital, le $2^{\text {ème }}$ trimestre sert de base de calcul dans la mesure où c'est à cette période de l'année que le niveau du prix des biens et des services est le plus élevé. Il faut par ailleurs noter que le coût de la vie (minimum vital) varie considérablement selon les différentes régions de la Russie, donc le montant de l'allocation également. Par exemple, dans la région de Penza ${ }^{8}$, le coût de la vie d'un enfant s'élève à 9817 roubles

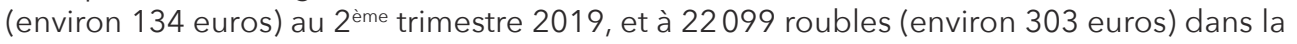
région de Kamtchatka?.

La procédure de financement de l'allocation prévue par la loi n 418 de 2017 est intéressante. Si l'allocation pour le premier enfant est financée par le budget fédéral de la Russie, l'allocation pour le deuxième enfant est en revanche payée aux frais du capital maternel de l'allocataire. Dans ce dernier cas, l'allocataire tire l'argent de sa propre poche et, après avoir fait la demande d'allocation, réduit mensuellement le montant de capital maternel qui lui est dû. Ainsi, l'État économise sur les paiements aux citoyens avec enfants. II semblerait néanmoins, au regard de la situation démographique actuelle, que le législateur puisse trouver d'autres sources d'économies budgétaires.

8 Décret du Gouvernement de la région de Penza du 9 août 2019 n473.

9 Décret du Gouvernement de la région de Kamtchatka du 29 juillet 2019 n³35-П. 


\section{FÉdérATION DE RUSSIE}

\section{III - LA RÉFORME DE 2019}

A l'initiative du Président (proposition exprimée dans le message du Président du 21 février 2019 au Parlement russe), le cercle des bénéficiaires de l'allocation familiale mensuelle a été élargi. En effet, le Président russe a proposé d'augmenter le "plafond " de revenu (quotient familial) donnant droit à une allocation, le faisant ainsi passer de 1,5 à 2 fois le minimum vital de la population active. Cette disposition devrait permettre de couvrir environ $70 \%$ des familles dans lesquelles un premier ou un deuxième enfants sont nés ${ }^{10}$. A ce titre, il faut en effet rappeler qu'en Russie, le nombre de pauvres est très élevé parmi les personnes ayant des enfants mineurs.

La proposition du Chef de l'Etat a bien évidemment reçu le soutien du Parlement et a été consacrée par la loi du 2 août $2019 n^{\circ} 305$, laquelle prévoit également une augmentation de la durée de versement de l'allocation familiale mensuelle - de 18 mois à 3 ans - à compter du 1 er janvier 2020.

L'ensemble des mesures ainsi prises ne peut être apprécié que positivement et entraînera peut-être un effet stimulant sur le taux de natalité dans le pays.

Cependant, force est de constater que le législateur ne prête son attention qu'aux enfants de moins de 3 ans. Concernant les enfants âgés de 3 à 18 ans, les prestations ne sont pas versées au niveau fédéral. Certes, une « allocation enfant » régionale est versée aux familles pauvres ayant un enfant de moins de 16 ans, mais son montant est généralement faible: par exemple, dans la région de Penza, il est de 308 roubles par mois (soit environ 4 euros). Enfin, dans certaines régions de la Russie, des allocations pour le troisième enfant et les enfants suivants sont parfois versées (à hauteur de 560 roubles par mois soit environ 7,70 euros).

Pour toutes ces raisons, il serait souhaitable que la prochaine étape de la réforme législative en matière de prestations familiales aboutisse à l'adoption de mesures d'aide financière pour toutes les familles ayant des enfants de moins de 18 ans, sans condition.

10 Message du Président de la Russie au Parlement: https://rg.ru/gazeta/2019/02/21.html 